Mittheilung aus dem Laboratorium des eidgenössischen

Polytechnicums Zürich.

(Eingelaufen den b. Mai 1891.)

\title{
Untersuchungen über Azole.
}

(Fortsetzung.)

14) Ueber Nitrosoverbindungen der Thiazolreihe; von Eduard Näf.

Die sogenannten Amidothiazole verhalten sich nach den Untersuchungen von Traumann*) und Wohmann**) als tautomere Verbindungen.

Aehnlich wie das sogenannte Cyanamid als echtes Cyanamid $\mathrm{C}_{\mathrm{NH}_{\mathbf{2}}}^{\mathrm{N}}$ und Carbodiimid $\mathrm{C}_{\mathrm{NH}}^{\mathrm{NH}}$ in Wirkung tritt, reagiren sie im Sinne der zwei Formeln :<smiles>Nc1nccs1</smiles>

$\mu$-Amidothiazol.
II. $\stackrel{\mathrm{HC}}{\mathrm{HC}}=\mathrm{NH}$

$\mu$-Imidothiazolin.

Nur ist immer deullicher hervorgetreten, dafs die zweite Formel mit symmetrischer Vertheilung der Wasserstoffatome an Stickstoff die begünstigtere Atomgruppirung darstellt, auf die sich fast alle Reactionen beziehen lassen. So treten bei der Einwirkung von Alkyljodiden zwei isomere monalkylirte Basen auf, welche sich nur von der zweiten Formel ableiten können, und von den dialkylirten sind nur die Dialkylimidothiazoline bekannt, während die echten Dialkylamidothiazole noch nicht dargestellt werden konnten.

Bei der Einwirkung von salpetriger Säure auf die ur-

*) Diese Annalen $\mathbf{2 4 9}, 31$.

**) Daselbst 259, 228. 
sprünglichen „Amidothiazole“ erhält man ebenfalls Substanzen, die man zwar als "Diazothiazolhydrate“ bezeichnen kann, die sich aber auch als Nitrosoimidothiazoline verhalten und thatsächlich zwischen echten Diazo- und echten Nitrosoverbindungen stehen.

Von diesen Körpern hatte der einfachste bisher noch nicht dargestellt werden können. Es ist mir indefs gelungen, aus Amidothiazol das Diazothiazolhydrat oder Nitrosoimidothiazolin wenigstens in ziemlich reinem Zustande zu erhalten.

Meine eigentliche Aufgabe bestand aber darin, die Producte der Einwirkung von salpetriger Säure auf monosubstituirte Basen zu untersuchen. Von denselben sind am besten bekannt die Methylirungsproducte des im Kern methylirten Amidothiazols, des sogenannten Rhodanpropimins*). Aber da schon die ursprüngliche Base, das Rhodanpropimin, gegen salpetrige Säure weit weniger glatt reagirt als das Amidothiazol selbst **), so wurden auch nicht die Alkylderivate des Rhodanpropimins auf ihr Verhalten gegenüber salpetriger Säure untersucht, sondern die des Amidothiazols, welche allerdings noch nicht dargestellt worden waren. Die erhaltenen Producte erwiesen sich, wie zu erwarten war, im allgemeinen als echte Nitrosoverbindungen; im speciellen zeigten sie freilich mehrere eigenthümliche Abweichungen.

Diazothiazolhydrat oder $\mu$-Nitrosoimidothiazolin,

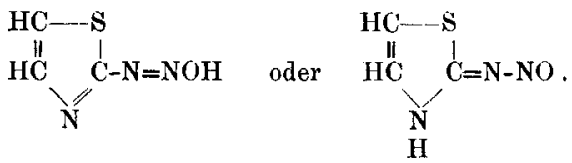

Diese Verbindung ist bereits von $\mathbf{S} \operatorname{ch}$ at $\left.\mathrm{z} \operatorname{man} \mathbf{n}{ }^{* * *}\right)$ be-

*) Ber. d. deutsch. chem. Ges. 20, 3118.

**) Diese Annalen 250, 277.

***) Daselbst 264, 9. 
obachtet worden, konnte aber von ihm nicht isolirt werden. Beim Einleiten von salpetriger Säure in eine mit Eis gekühlte Lösung von salzsaurem Amidothiazol erschienen zwar orangerothe Flocken, allein dieselben zersetzten sich sehr rasch unter Gasentwicklung. Besser gelangt man zum Ziele, wenn man nach meinen bei den methylirten Basen gemachten Erfahrungen in concentrirter Lösung und zwar folgendermafsen arbeitet :

Salpetersaures Amidothiazol - durch Auflösen von Amidothiazol in verdünnter Salpetersäure in grofsen, weifsen Krystallen erhalten - wird in möglichst wenig Wasser gelöst, die Lösung mit Eis und Kochsalz bis auf $-10^{\circ}$ abgekühlt uud nun tropfenweise mit einer ebenfalls gekühiten, ganz concentrirten Lösung von Natriumnitrit versetzt. Die Flüssigkeit färbt sich erst gelb, dann braun und scheidet nach einigen Minuten, besonders beim Schütteln oder Umrühren, in reichlicher Menge orangerothe Flocken ab. Die Fällung mufs unter Eiskühlung filtrirt, möglichst schnell auf einer Thonplatte abgeprefst und im Exsiccator getrocknet werden. Auch ist es nicht möglich, sie weiter zu reinigen. Wiederholte Analysen des direct erhaltenen Productes ergaben zwar stets mehr als ein Procent Stickstoff zu wenig, erwiesen indefs doch zugleich im Verein mit seinem chemischen Verhalten, dafs es nichts anderes sein konnte, als ein oberflächlich zersetztes Diazothiazolhydrat.

I. $0,1102 \mathrm{~g}$ Substanz ergaben 30,9 cbcm Stickgas bei $18^{\circ}$ und $724 \mathrm{~mm}$.

II. $0,0976 \mathrm{~g}$ Substanz gaben $28,0 \mathrm{cbcm}$ Stickgas bei $22^{\circ}$ und $724 \mathrm{~mm}$.

1Ir. $0,1246 \mathrm{~g}$ Substanz gaben 34,6 cbem Stickgas bei $14^{0}$ und $726 \mathrm{~mm}$.

IY. 0,0799 g Substanz gaben 22,2 cbem Stickgas bai $14^{\circ}$ und $727,5 \mathrm{~mm}$.

Berechnet für $\mathrm{C}_{8} \mathrm{H}_{3} \mathrm{~N}_{3} \mathrm{SO}$

$\mathbf{N}$
32,56

\begin{tabular}{|c|c|c|c|}
\hline \multicolumn{4}{|c|}{ Gefunden } \\
\hline I. & II. & III. & [V. \\
\hline 30,81 & 30,90 & 31,18 & $31,28$. \\
\hline
\end{tabular}

Diazothiazolhydrat bildet ein amorphes, orangerothes 
Pulver, ist löslich in Alkohol und Aether, unlöslich in Wasser. In feuchtem Zustande zersetzl es sich äufserst leicht, aber auch getrocknet verändert es sich nach und nach unter Schwärzung.

Langsam erhitzt, verkohlt es allmählich; taucht man aber die im Haarröhrchen befindliche Substanz in die bereits über $130^{\circ}$ erhitzte Schwefelsäure und erwärmt rasch höher, so zersetzt sie sich ziemlich scharf bei $140^{\circ}$ unter Verpuffung.

Diazothiazolhydrat wird selbst von verdünnten Säuren nur unter Zersetzung, bezw. Gasentwicklung und unter Bildung harzartiger Producte gelöst; dafs es von concentrirter Salzsäure und Bromwasserstoffsäure unter Stickstoffentwicklung in Chlor- und Bromthiazol übergeführt wird, ist bereits von $S c h$ a $\mathbf{z m a n n} \mathbf{n}^{*}$ ) mitgetheill worden.

Es scheint also die neue Verbindung in der Mitte zu stehen zwischen Diazo- und Nitrosokörpern. Die Spaltung durch concentrirte Halogenwasserstoffsäuren spricht für die erstere, diejenige durch verdünnte Säuren für die letztere Annahme. Vielleicht könnte man hier und überhaupt im Allgemeinen annehmen, dafs die Producte der Einwirkung von salpetriger Säure auf Amidothiazole in fester Form als Nitrosoverbindungen, in saurer Lösung aber als Salze höchst zersetzlicher Diazoverbindungen bestehen.

\section{Methylirte Imidothiazoline und ihre Nitrosoderivate.}

Was von Traumann **) für das Rhodanpropimin nachgewiesen worden ist, gilt auch für das Amidothiazol. Es existiren zwei isomere Monomethylderivate, die sich vom Imidothiazolin ableiten :

*) Diese Annalen 261,9 und $f$.

**) Daselbst $\mathbf{2 4 9}, 43$. 


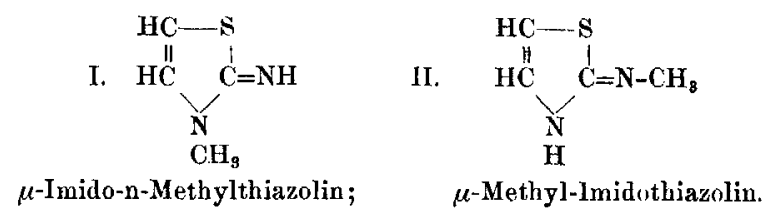

Das erstere, im Kern methylirte Product entsteht durch Einwirkung von Jodmethyl auf Imidothiazolin (Amidothiazol), das letztere durch Einwirkung von Monomethylthioharnstoff auf Dichloräther.

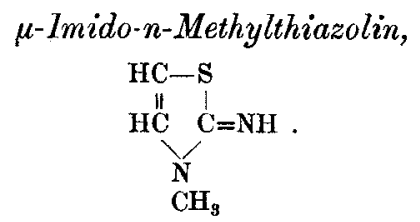

Das Amidothiazol oder Imidothiazolin reagirt erheblich schwerer mit Jodmethyl als sein $\alpha$-Methylderivat, das Rhodanpropimin. Es mufs mit etwas mehr als der theoretischen Menge Jodmethyl und wenig Methylalkohol im Rohr eingeschlossen und mehrere Stunden auf $130^{\circ}$ erhitzt werden. Der Röhreninhalt, beim Erkalten in schönen, langen Krystallen erstarrend, wird mit wenig kaltem Wasser behandelt, wobei das jodwasserstoffsaure Salz des Monomethylimidothiazolins in Lösung geht und die Hauptmasse der unveränderten Base zurückbleibt. Von dem in kleiner Menge entstandenen Salz der Dimethylbase wird das monomethylirte Product durch fractionirte Krystallisation befreit. Das reine, jodwasserstoffsaure Salz des $\mu$-Imidomethylthiazolins bildet weifse, luftbeständige Blätter vom Schmelzpunkt $175^{\circ}$, die sich nach und nach bräunen.

$0,3023 \mathrm{~g}$ lufttrockene Substanz ergaben $0,1590 \mathrm{~g} \mathrm{~J}$.

$$
\begin{array}{ccc} 
& \text { Berechnet fuir } & \text { Gefunden } \\
\mathrm{C} & \mathrm{C}_{4} \mathrm{~N}_{2} \mathrm{SH}_{6} \cdot \mathbf{H J} & \\
522,48 & 52,59 .
\end{array}
$$

Die aus dem Salze mittelst Kali abgeschiedene Base ist ein hellbraunes, äufserst hygroscopisches, im Vacuum nicht 
erstarrendes 0el von stark alkalischer Reaction. Sie löst sich in Säuren zu wohlcharakterisirten Salzen; das salzsaure Salz krystallisirt aus Wasser in grofsen, nicht hygroscopischen, aber sehr leicht löslichen Tafeln vom Schmelzpunkt $97^{\circ}$; das Platinsalz ist schwer löslich.

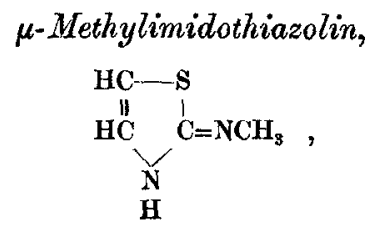

entsteht bei Einwirkung von Monomethylthioharnstoff - aus alkoholischem Ammoniak und Methylsenföl in prachtvollen Krystallen erhaiten - auf Dichloräther :

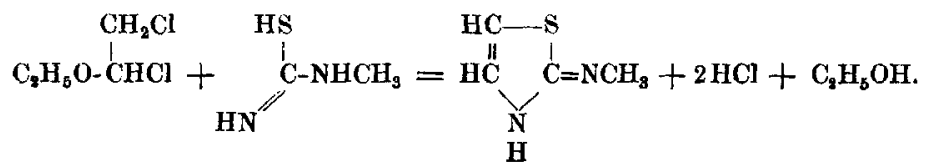

Monomethylthioharnstoff wird in Wasser gelöst und mit der berechneten Menge Dichloräther am Rückflufskühler erwärmt, bis der Geruch nach Chloracetaldehyd verschwunden ist. Dann wird eingedampft, alkalisch gemacht und mit Aether ausgezogen; der ätherische Extract wird wieder mit Salzsäure ausgeschüttelt und schliefslich die Base nochmals durch Kali abgeschieden. Sie bildet ein unangenehm riechendes, hellbraunes Oel, das bei längerem Stehen in luftverdünnten Raum über Schwefelsäure zu spitzen Nadeln erstarrt, die aber äufserst hygroskopisch sind; daher wurde zur Analyse das salzsaure Salz verwendet, das aus Wasser als undeutlich krystallisirte Masse erhalten wird und bei 79 bis $80^{\circ}$ schmilzt. Es ist ebenfalls hygroskopisch, aber nicht in so hohem Grade, wie die freie Base.

$0,1253 \mathrm{~g}$ im Exsiccator getrocknete Substanz gaben 0,0296 Cl. Annalen der Chemie 465. Bd. 


$\begin{array}{ccc} & \text { Berechnet tür } & \text { Gefunden } \\ \mathrm{C}_{4} \mathrm{~N}_{2} \mathrm{SH}_{6} \mathrm{HCl} & \\ \text { C1 } & 23,59 & 23,62 .\end{array}$

Platinchlorid bildet ein in langen, spiefsigen Nadeln krystallisirendes Doppelsalz, das nicht weiter untersucht wurde.

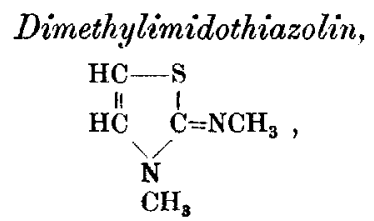

entsteht nicht nur durch Methylirung aus beiden eben besprochenen Monomethylbasen, sondern auch, und zwar viel bequemer, durch Einwirkung von Dimethylthioharnstoff - nach Andreasch's *) Angabe aus Methylamin und Methylsenfōl erhalten - auf Dichloräther, wodurch zugleich bewiesen wird, dafs alle Alkylderivate des sogenannten Amidothiazols auf das Imidothiazolin zurückzuführen sind. Die Reaction verläuft analog der Bildung von $\mu$-Methylimidothiazolin.

Man erwärmt die wässerige Lösung des Dimethylthioharnstoffes mit der berechneten Menge Dichloräther, dampft hierauf ein und scheidet aus dem so erhaltenen salzsauren Salz die freie Base durch Kali ab. Dieselbe zeigt die nämljchen Eigenschaften wie die Monomethylbasen. Sie ist ölig und hygroskopisch, liefert aber gut krystallisirende Salze.

Das salzsaure Salz, welches bei $222^{\circ}$ schmilzt, wurde der Analyse unterworfen.

0,1795 g Substanz gaben $0,0384 \mathrm{Cl}$.

$$
\begin{array}{ccc} 
& \text { Berechnet für } & \text { Gefunden } \\
\text { CI } & \mathrm{C}_{5} \mathrm{~N}_{\mathbf{8}} \mathrm{SH}_{8} \mathrm{HCl} & \\
& 21,58 & 21,39 .
\end{array}
$$

Verhalten der Monomethylimidothiazoline gegen Salzsäure. - Aus dem Verhalten der Monomethylderivate des

*) Monatshefte für Chemie $2,277$. 
Rhodanpropimins gegen Salzsäure bei höherer Temperatur hatte Traumann*) die Constitution derselben bestimmt. Hierbei wird nämlich die stickstoffhaltige Gruppe der Seitenkette abgespalten, im einen Fall als Ammoniak, im anderen als Methylamin.

Auch diese einfachsten methylirten Producte verhalten sich ganz analog; die durch Jodmethyl aus Amidothiazol erhaltene Base liefert Ammoniak und solite in den StickstoffMethyläther des Ketothiazolins übergehen :

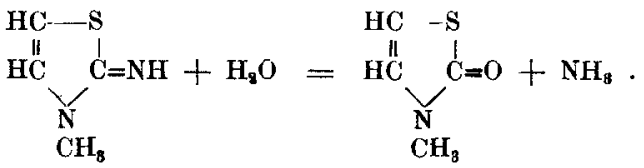

Die aus Monomethylthioharnstoff erzeugte Base spaltet Methylamin ab und sollte $\mu$-Oxythiazol (Ketothiazolin) liefern :

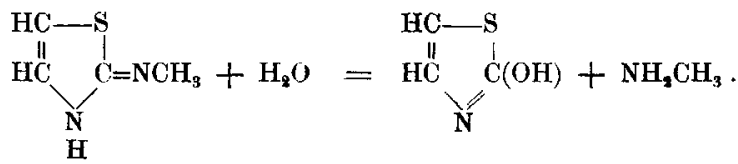

Aber auch hier konnten diese sauerstoffhaltigen Thiazolderivate nicht isolirt werden. Bei Temperaturen unter $230^{\circ}$ wirkte die concentrirte Salzsäure überhaupt nicht ein; überschritt man dagegen diese Grenze, so schien auch der Thiazolkern gesprengt worden zu sein, da nur Schwefelwasserstoff und harzartige Producte entstanden waren.

Verhalten der Methylimidothiazoline gegen salpetrige Säure. - Wie zu erwarten war, reagiren die beiden Monomethylbasen wie secundäre Anine und liefern, am besten durch Umsetzung in schwach salzsaurer Lösung mit einer concentrirten Lösung von Natriumnitrit, zwei isomere Nitrosamine :

*) Diese Annalen $\mathbf{2 0} 4 \mathbf{4}, 49$. 
I. $\stackrel{\mathrm{HC}}{\mathrm{H}} \stackrel{\mathrm{C}}{\mathrm{C}}=\mathrm{N}-\mathrm{NO}$

n-Methyl- $\mu$-Nitrosoimidothiazolin
II. $\stackrel{\mathrm{HC}}{\mathrm{HC}} \stackrel{\mathrm{C}}{\mathrm{C}=\mathrm{NCH}_{3}}$

n-Nitroso- $\mu$-Methylimidothiazolin.

Die Dimethylbase ist indifferent gegenüber salpetriger Säure; sie besitzt also, abgesehen von ihrer tertiären Natur, keine Aehnlichkeit mehr mit dem Dimethylanilin, d. i. die an Kohlenstoff gebundenen Wasserstoffatome des Thiazolkernes sind nicht, wie die des Benzolkernes, durch die Nitrosogruppe vertretbar. Auch dadurch wird bestätigt, dafs in obigen Nitrosaminen die Nitrosogruppe nur an Stelle des Imidwasserstoffes getreten sein kann und dafs denselben wirklich die angegebene Constitution zukommt.

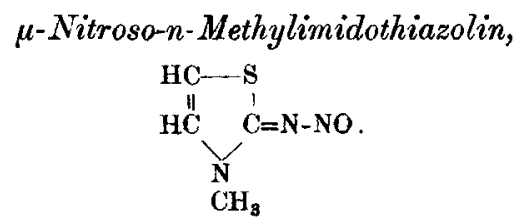

Die durch Methylirung aus Amidothiazol erhaltene Base Jäfst sich nur unter $0^{0}$ und nur in concentrirter Lösung glatt nitrosiren.

Salzsaures $\mu$-Imidomethylthiazolin wird in wenig Wasser gelöst und mit einigen Tropfen verdünnter Salzsäure versetzt. Dann wird die Lösung auf $-5^{0}$ bis $-10^{\circ}$ abgekühlt, wobei sie gewöhnlich erstarrt und nun mit einer ganz concentrirten Lösung von Natriumnitrit vermischt. In dem Mafse, wie die gefrorene Musse aufthaut, fällt der Nitrosokörper in Blättchen heraus. Der so dargestellte, abgesaugte, mit Wasser ausgewaschene und rasch auf dem Thonteller getrocknete Körper ist bereits chemisch rein. Er bildet goldglänzende, gelbe Blatter, die nur einen schwachen, nicht sonderlich charakteristischen Geruch besitzen und bei $161^{\circ}$ unter geringer Ver- 
puffung schmelzen. Sie sind löslich in Alkohol und Aether, nur wenig in Wasser.

I. $0,1010 \mathrm{~g}$ Substanz gaben $25,7 \mathrm{cbcm}$ Stickgas bei $10^{\circ}$ und $730 \mathrm{~mm}$.

II. $0,1210 \mathrm{~g}$ Substanz gaben $31,4 \mathrm{ebcm}$ Stickgas bei $14^{\circ}$ und $731 \mathrm{~mm}$.

\begin{tabular}{cccc} 
& $\begin{array}{c}\text { Berechnet für } \\
\mathrm{C}_{4} \mathrm{~N}_{3} \mathrm{H}_{5} \mathrm{SO}\end{array}$ & \multicolumn{2}{c}{ Gefunden } \\
$\mathrm{N}$ & 29,37 & $\mathrm{II}$. \\
29,25 & $29,35$.
\end{tabular}

Das $\mu$-Nitrosomethylimidothiazolin ist, einmal isolirt, ziemlich beständig und vor allem im trockenen Zustande unveränderlich. In Alkalien löst es sich auffallenderweise unverändert auf, wobei vielleicht salzartige Verbindungen von der Formel :<smiles>Cn1ccsc1=NN(O)O</smiles>

entstehen.

In seinem übrigen Verhalten ist es ein echtes Nitrosamin, was gegenüber dem später $z u$ besprechenden Isomeren besonders zu betonen ist. Wird es zum Beispiel mit Salzsäure von beliebiger Stärke übergossen, so entweicht, indem es sich auflöst, massenhaft salpetrige Säure und aus der Mutterlauge krystallisirt in grofsen Platten ein salzsaures Salz, das umkrystallisirt den Schmelzpunkt $\mathbf{9 7}^{\circ}$ des salzsauren n-Methylimidothiazolins besitzt. Es wird also die ursprüngliche Base zurückgebildet. In ähnlicher Weise verläuft die Spaltung bei Anwendung von Brom- und Jodwasserstoffsäure, Schwefelsäure, Essigsäure, ebenso bei der Einwirkung starker Reductionsmittel, wie schwefliger Säure, Zink- und Schwefelsäure.

Schwache Reductionsmittel dagegen veranlassen auch hier die Bildung eines Hydrazins. 


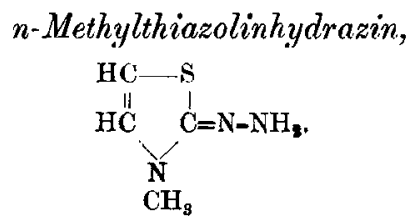

Dasselbe entsteht freilich nur in sehr geringer Menge, am besten noch, wenn man folgendermafsen verfährt :

n-Methyl- $\mu$-Nitrosoimidothiazolin wird in wenig Alkohol gelöst, zuerst mit überschüssigem Zinkstaub und dann allmählich unter Kühlung tropfenweise mit Eisessig versetzt. Nach zwei Stunden wird die vollkommen entfärbte Lösung filtrirt, der Alkohol abgedampft und der Rückstand unter Kühlung mit ganz concentrirter Kalilauge versetzt. Die dadurch abgeschiedene Hydrazinbase macht sich sofort durch einen äufserst widrigen Geruch bemerkbar. Sie wird in Aether aufgenommen und hinterbleibt beim Verdunsten desselben als gelbgrünes Oel, dem stets ein wenig zurückgebildetes n-Methylimidothiazolin beigemengt ist. Um dasselbe zu entfernen, löst man das Oel in concentrirter Salzsäure, wobei das salzsaure Salz des Hydrazins in hellgelben, glänzenden Nädelchen auskrystallisirt.

Die aus diesem reinen Salz abgeschiedene Base ist etwas heller gefärbt, behält aber den unangenehmen Geruch bei. Sie bildet ein schwer lösliches Pikrat, welches in gelbgrünen Nadeln ausfältt, und giebt als echte Hydrazinbase mit Aldehyden und Ketonen krystallinische Condensationsproducte.

Wegen der äufserst schlechten Ausbeute und der öligen Beschaffenheit der freien Base wurde nur der Chlorgehalt des salzsauren Salzes durch Titration bestimmt.

I. $0,1190 \mathrm{~g}$ Substanz gaben 0,02556 Cl.

II. $0,0254 \mathrm{~g} n$ n 0,00543 ,

\begin{tabular}{cccc} 
Berechnet für & \multicolumn{2}{c}{ Gefunden } \\
$\mathrm{C}_{4} \mathrm{~N}_{9} \mathrm{H}_{8} \mathrm{SCl}$ & $\overbrace{1 .}$ & II. \\
21,45 & 21,48 & $21,30$.
\end{tabular}

Die .Reduction des Nitrosokörpers in alkalischer, bezw. ammoniakalischer Lösung ergab eine noch schlechtere Aus- 
beute. Die Flüsslgkeit entfärbt sich, unter Kühlung mit Zinkstaub versetzt, zwar sehr rasch, aber das mit Aether ausgeschüttelte Filtrat enthält stets in gröfserer Menge das n-Methylimidothiazolin als die Hydrazinbase.

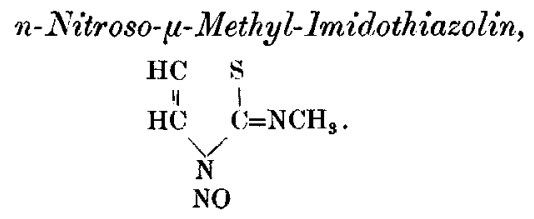

Die in der Seitenkette methylirte Base aus Monomethylthioharnstoff und Dichloräther läfst sich etwas leichter nitrosiren als die eben besprochene, im Kern methylirte; im übrigen aber unter den gleichen Bedingungen.

Zu der concentrirten, mit Eis und Kochsalz gekühlten, wässerigen Lösung des salzsauren Salzes wird eine ebenso dargestellte Lösung von Natriumnitrit tropfenweise zugefügt.

Der dem oben besprochenen isomere Nitrosokörper scheidet sich hier nicht allmählich, sondern unter erheblicher Erwärmung plötzlich ab; namentlich beim Kratzen der Gefäfswände mit einem Glasstab erstarrt die Flüssigkeit sehr rasch zu einem Brei gelblich-weifser, kleiner Krystalle, die einen charakteristischen Geruch, ähnlich wie Acetamid, besitzen. Die Mutterlauge wird rasch abgesaugt, die Krystalle werden mit Wasser gewaschen, auf dem Thonteller abgeprefst und über Chlorcalcium getrocknet.

1. $0,1143 \mathrm{~g}$ Substanz gaben 29,5 ebcm Stickgas bei $13^{0}$ und $726 \mathrm{~mm}$.

1]. $0,1000 \mathrm{~g}$ Substanz gaben 26,3 cbcm Stickgas bei $18^{\circ}$ und $722 \mathrm{~mm}$.

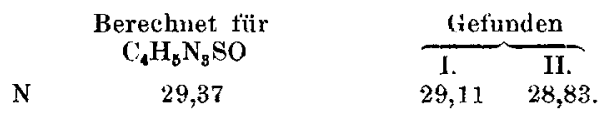

Aus verdünnter Lösung erhält man überhaupt keine Fällung und bei Unterlassung des Kühlens nur ein Oel, das zu einer harzigen Masse erhärtet und hauptsächlich aus Zersetzungsproducten des Nitrosokörpers zu bestehen scheint. 
Das im Kern nitrosirte, in der Seitenkette methylirte Imidothiazolin verhält sich in mehreren Punkten anders als sein Isomeres und als die übrigen Nitrosamine.

Erstens ist es viel zersetzlicher als sein Isomeres, erimnert also in dieser Hinsicht viel mehr an die Diazoverbindungen, beziehungsweise an die Producte aus salpetriger Säure und nicht methylirten Amidothiazolen. Auch in vollständig trockenem Zustand zersetzt es sich bereits bei gewöhnlicher Temperatur allmählich unter Bräunung. Durch Wasser wird es bei längerem Kochen verharzt, durch Alkohol zwar ziemlich leicht gelöst, aber ebenfalls zum Theil in schmierige Producte verwandelt; nur aus Aceton kann es vorsichtig unverändert umkrystallisirt werden.

Der Nitrosokörper schwärzt sich beim langsamen Erhitzen und schmilzt bei etwa $140^{\circ}$ unter völliger Zersetzung; bei raschem Erhitzen verpufft er zwischen $110^{\circ}$ und $120^{\circ}$; ein scharfer Zersetzungspunkt konnte nie beobachtet werden.

Wässeriges und alkoholisches Ammoniak, sowie Alkalien lösen ihn leicht auf. Hierbei wird er, wie die lebhafte Gasentwicklung andeutet, zum gröfsten Theil vollständig zersetzt, zum Theil aber in methylirte Base zurückverwandelt, die als solche durch die nochmalige Ueberführung in den ursprünglichen Nitrosokörper erkannt wurde. Die Nitrosirung ist also zugleich eine sehr empfindliche Reaction auf kleine Mengen von $\mu$-Methylimidothiazolin.

Noch auffallender als die Unbeständigkeit ist das

Verhalten des $\mu$-Methyl-n-Nitrosoimidothiazolins gegenüber Reductionsmitteln.

Gegen die Erwartung konnte hierbei weder Bildung eines Hydrazins noch Rückverwandlung in das ursprüngliche $\mu$-Methylimidothiazolin beobachtet werden. Selbst bei vorsichtigster Behandlung mit Zinkstaub und Eisessig in alkoholischer Lösung wurde die Flüssigkeit zwar momentan entfärbt, nahm aber 
beim Abdampfen des überschüssigen Alkohols eine intensiv rothe Farbe an und ergab bei Zusatz von festem Kali nur massenhaft Ammoniak, im ätherischen Extract aber überhaupt kein basisches Reactionsproduct.

Reduction in alkalischer Lösung ist unmöglich, da, wie oben angegeben, der Nitrosokörper schon durch Berührung mil Alkalien verändert wird.

Am eigenthümlichsten ist das

Verhalten dieses Nitrosokörpers gegen Säuren.

Sämmtliche Mineralsäuren und selbst Essigsäure wirken spaltend ein; doch nur bei Anwendung von verdünnter Schwefelsäure oder Salzsäure gelang es, eine kleine Menge der Base zurückzuerhalten, also einfach die Nitrosogruppe durch Wasserstoff zu ersetzen.

Ganz vorwiegend bildet sich bei Anwendung von Halogenwasserstoffsäuren ein anderes Product, welches ein besonderes Interesse verdient. Dasselbe ist ein Salz einer im freien $\mathrm{Zu}$ stande sehr zersetzlichen Base von der Formel $\mathrm{C}_{4} \mathrm{H}_{3} \mathrm{~N}_{3} \mathrm{~S}$, welche aus dem ursprünglichen Nitrosokörper $\mathrm{C}_{4} \mathrm{H}_{5} \mathrm{~N}_{3} \mathrm{SO}$ durch Abspaltung von Wasser entstanden sein muls :

$$
\mathrm{C}_{4} \mathrm{H}_{5} \mathrm{~N}_{3} \mathrm{SO}=\mathrm{C}_{4} \mathrm{H}_{3} \mathrm{~N}_{3} \mathrm{~S}+\mathrm{H}_{2} \mathrm{O} \text {. }
$$

Diese Reaction ist in Anbetracht der Structurformel des Nitrosamiıs nur in einer einzigen Weise denkbar : dafs sich das Sauerstoffatom der Nitrosogruppe mit zwei Wasserstoffatomen des Methyls verbindet und dafs dadurch das betreffende Kohlenstoffatom mit dem betreffenden Stickstoffatom in Beziehung tritt :<smiles>C=CN=C1SC=CC1=[N+]=O</smiles>

Danach ist die neue Base auf ähnliche Weise gebildet, wie die sogenannten Anhydrobasen der aromatischen Diamine und enthält also neben dem Thiazolkern einen an denselben 
angeschlossenen Ring von 2 Kohlenstoff- und 3 Stickstoffatomen, welcher von Bla din ${ }^{*}$ ) als Triazol bezeichnet wurde und in den Derivaten des sogenannten Cyananilins und Dicyanphenylhydrazins enthalten ist.

Diese Base mag daher, obgleich nicht ganz correct, bezeichnet werden als

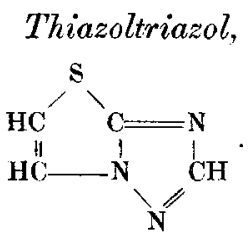

Die Umstände, unter welchen das Thiazoltriazol aus dem Nitrosamin durch intramolekulare Wasserabspaltung entsteht, erscheinen etwas auffallend; man kennt indessen bereits zwei Reactionen, welche auf ganz ähnliche Weise erklärt werden müssen :

Der von $\mathrm{F}$ is ch er und $\mathrm{Hep} \mathrm{p}^{* *}$ ) beobachtete Uebergang des $\alpha$-Nitroso- $\beta$-Aethylnaphtylamins in Aethenyl- $\alpha-\beta$-Naphtylendiamin
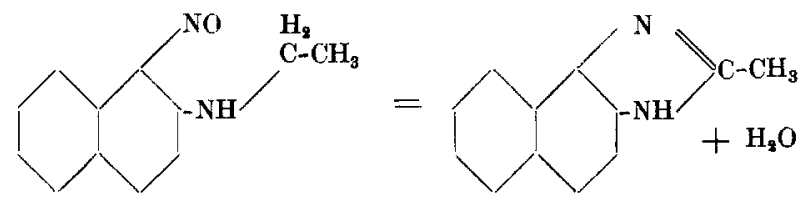

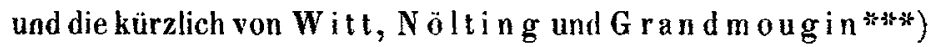
entdeckte Umwandlung der Diazoverbindung des $m$-Nitroorthotoluidins in $\mathbf{m}$-Nitroindazol :

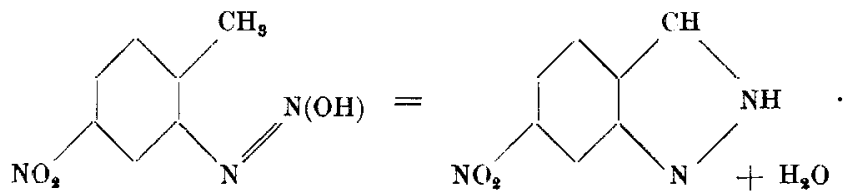

Auch vollziehen sich diese Reactionen unter ganz ähn-

*) Ber. d. deutsch. chem. Ges. 23, 3785.

**) Daselbst 20, 1247, 2471.

**) Daselbst 28, 3643 . 
lichen Bedingungen, wenigstens bei den Nitrosaminen. Fis c h e $\mathrm{r}$ und $\mathrm{H} \mathrm{epp}$ lösten das Nitrosamin in Alkohol, setzten alkoholische Salzsäure zu und erhielten nach längerem Stehen einen Krystallbrei des salzsauren Salzes der Anhydrobase.

Auch das vorliegende Nitrosamin verhält sich unter denselben Bedingungen ähnlich, doch verfährt man besser folgendermafsen :

Frisch bereitetes n-Nitroso- $\mu$-Methylimidothiazolin wird mit gekühller 10 procentiger Salzsäure übergossen. Dabei entweicht nur in geringer Menge salpetrige Säure - entsprechend der kleinen Menge zurückgebildeter Base. Die Lösung wird hierauf erwärmt, bis keine Gasentwicklung mehr zu heobachten ist und dann mehrere Stunden sich selbst überlassen. Alsdann erfüllt sie sich mit schönen, nadelförmigen Krystallen. Abgeprefst und mit wenig Alkohol und Aether gewaschen sind sie, wenn aus reinem Nitrosokörper bereitet, vollständig rein. Sie bräunen sich schon bei $130^{\circ}$, schmelzen aber erst bei 210 bis $220^{\circ}$.

Sie sind zufolge der Analyse das mit zwei Molekülen Wasser krystallisirende

$$
\begin{gathered}
\text { salzsaure Salz des Thiazoltriazols: } \\
\qquad \mathrm{C}_{4} \mathrm{~N}_{3} \mathrm{H}_{3} \mathrm{~S} . \mathrm{HCl}+2 \mathrm{H}_{2} \mathrm{O} .
\end{gathered}
$$

J. $0,1786 \mathrm{~g}$ Substanz lieferten $0,1591 \quad \mathrm{CO}_{2}$ und $0,0733 \mathrm{H}_{2} \mathrm{O}$.

II. $0,1543 \mathrm{~g} n \pi 0,1842 \mathrm{BaSO}_{4}$.

1II. a) $0,1019 \mathrm{~g}$ Substauk gaben 19,7 cben Stickgas bei $16^{\circ}$ und $714 \mathrm{~mm}$.

b) $0,1014 \mathrm{~g}$ Substanz gabor 19,4 cbem Stickgas bei $16^{\circ}$ und $725 \mathrm{~mm}$.

IV. a) $0,1006 \mathrm{~g}$ substanz lieferten $0,01811 \mathrm{Cl}$.

\begin{tabular}{|c|c|c|c|}
\hline & \multirow{2}{*}{$\begin{array}{l}\text { Berechnet für } \\
\mathrm{C}_{4} \mathrm{~N}_{3} \mathrm{H}_{8} \mathrm{SClO}_{8}\end{array}$} & \multicolumn{2}{|c|}{ Gefunden } \\
\hline & & I. & II. \\
\hline$c$ & 24,3 & 24,3 & - \\
\hline & 4,05 & 4,56 & - \\
\hline & 16,20 & 16,4 & - \\
\hline & 21,26 & 21,21 & 21,25 \\
\hline & 17,97 & 18,00 & 18,08 \\
\hline
\end{tabular}

b) $0,0918 \mathrm{~g}, \pi 0,0166$ n

Das Krystallwasser ist ziemlich fest gebunden; das Salz 
verwittert nicht über Schwefelsäure, sondern giebt das Wasser erst zwischen 130 und $140^{\circ}$ ab.

$0,2650 \mathrm{~g}$ Substanz verloren $0,0490 \mathrm{H}_{2} \mathrm{O}$.

$$
\begin{array}{ccc} 
& \text { Berechnet für } & \text { Gefunden } \\
& \mathrm{C}_{4} \mathrm{H}_{4} \mathrm{~N}_{3} \mathrm{SCl} .2 \mathrm{H}_{8} \mathrm{O} & \\
\mathrm{H}_{2} \mathrm{O} & 18,23 & 18,49 .
\end{array}
$$

Dabei hatte sich die Substanz allerdings stark gebräunt, aber trotzdem nur ganz oberflächlich zersetzt. Sie löste sich nicht nur in angesäuertem Wasser ganz klar, sondern zeigte auch noch annähernd den für wasserfreies Salz berechneten Chlorgehalt. Eine Chlorbestimmung nach Volhard ergab :

$$
\begin{array}{ccc} 
& \text { Berechnet für } & \text { Gefunden } \\
\mathrm{Cl} & \mathrm{C}_{4} \mathrm{H}_{4} \mathrm{~N}_{\mathrm{s}} \mathrm{ClS} & \\
\mathrm{Cl} & 21,98 & 21,24 .
\end{array}
$$

Wie schon erwähnt, wirkt auch Bromwasserstoffsäure ganz analog auf den Nitrosokörper.

Bromwasserstoffsaures Thiazoltriazol.

Aus der vorsichtig bereiteten bromwasserstoffsauren Lösung scheidet es sich längerem Stehen in prachtvollen, langprismatischen Krystallen ab, die getrocknet analysenrein sind.

Das Salz enthält ebenfalls zwei Moleküle Krystallwasser, die es bei 130 bis $140^{\circ}$ verliert.

$0,2629 \mathrm{~g}$ Substanz verloren $0,0382 \mathrm{H}_{2} \mathrm{O}$.

$\begin{array}{ccc} & \text { Berechnet für } & \text { Gefunden } \\ \mathrm{H}_{2} \mathrm{O} & \mathrm{C}_{4} \mathrm{H}_{4} \mathrm{~N}_{3} \mathrm{SBr} .2 \mathrm{HO}_{2} & \\ 14,87 & 14,53 .\end{array}$

$0,1143 \mathrm{~g}$ Substanz gaben 18 cbem Stickgas bei $16^{\circ}$ und $726 \mathrm{~mm}$. $0,0806 \mathrm{~g} n, 0,0268 \mathrm{Br}$.

$\begin{array}{lcc} & \text { Berechnet für } & \text { Gefunden } \\ \mathrm{N} & \mathrm{C}_{4} \mathrm{~N}_{3} \mathrm{H}_{8} \mathrm{SBrO}_{7} & \\ \mathrm{Br} & 17,35 & 17,52 \\ & 33,06 & 33,25 .\end{array}$

Das bromwasserstoffsaure Salz bräunt sich nach längerer Zeit schon bei gewöhnlicher 'Temperatur, rasch beim Trocknen bei $140^{\circ}$; langsam höher erhitzt, verkohlt es, ohne zu schmelzen.

Das freie Thiazoltriazol kann aus den Salzen nur durch ganz concentrirte Pottaschelösung in Form von Flocken abgeschieden, aber kaum vollig rein erhalten werden. Werden 
die Flocken abgeprefst und von dem anhaftenden Kaliumcarbonat durch inehrmaliges Umkrystallisiren aus Methylalkohol - in Aether ist die Base unlöslich - befreit, so resultirt ein braungefärbtes, harzartiges $0 \mathrm{el}$, das nach längerem Stehen im luftverdünnten Raum glasartig erstarrt und sich in Salzsäure wieder zu dem mit zwei Molekülen Wasser krystallisirenden Chlorhydrat auflöst. Die Base ist sehr leicht löslich in Wasser, hat alkalische Reaction und reducirt Fehling'sche Lösung. Schüttelt man die wässerige Lösung des salzsauren Salzes mit feuchtem Silberoxyd, so geht die Base leicht in das Filtrat vom Chlorsilber, giebt aber beim Eindampfen denselben Syrup und ist also auch auf diese Weise nicht rein zu gewinnen, weshalb von der Analyse derselben abgesehen wurde.

Die Versuche, die Constitution der Anhydrobase durch ihre Umsetzungsproducte noch eingehender zu begründen, ergaben nur in einem Fall ein befriedigendes Resultat. Weder durch Reduction in saurer oder in alkalischer Lösung, noch durch die Einwirkung von Salzsäure bei höherer Temperatur kann man andere basische Producte nachweisen als Ammoniak; die Haupimasse der Substanz geht in intensiv roth gefärbte, aber nicht falsbare Zersetzungsproducte über. Ebenso wird sie durch Kaliumpermanganat völlig verbrannt.

Auffallender Weise wird jedoch bei Einwirkung von concentrirter Natriumnitritlösung auf die wässerige Lösung des salzsauren Thiazoltriazols in grofser Menge das n-Nitroso$\mu$-Methylimidothiazolin zurückgebildet. Es wird also hier, umgekehrt wie bei der Bildung der Base, der King wieder geöfnet und damit wenigstens die nahe Beziehung zwischen Anhydrobase und ursprünglichem Nitrosokörper dargethan.

Phenylirte Imidothiazoline und deren Nitrosoderivate.

Diese Verbindungen wurden vor allem deshalb untersucht, weil das Verhalten derselben einen indirecten Beweis dafür liefern konnte und geliefert hat, dafs die Constitution des Thia- 
zoltriazols und seine Bildung aus dem im Kern nitrosirten Methylimidothiazolin richtig gedeutet worden ist. Denn wenn hierbei aus dem letztern die zwei Wasserstoffatome der Nethylgruppe entfernt worden sind, so kann das entsprechende phenylirte Nitrosamin

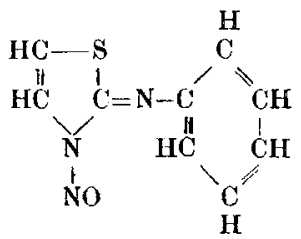

keine entsprechende Anhydrobase bilden, weil ihm eben zwei an ein und dasselbe Kohlenstoffatom gebundene Wasserstoffatome fehlen, welche zur Ringbildung nothwendig sind.

$\mu$-Phenylimidothiazolin<smiles>[AsH2]c1ccc[nH]1</smiles>

entsteht aus Dichloräther und Monophenylthioharnstoff auf dieselbe Weise, indefs nicht so glatt, wie die methylirte Base aus Monomethylthioharnstoff. Aus dem sehr hygroskopischen salzsauren Salz durch Kali abgeschieden, krystallisirt sie aus Alkohol, Aether und Benzol in kleinen, weifsen Nadelı vom Schmelzpunkt 124". Die Salze krystallisiren schlecht; die Analyse der freien Base ergab :

aus $0,0994 \mathrm{~g}$ Substanz 15 ebcm Stickgas bei $22^{\circ}$ und $724 \mathrm{~mm}$.

$$
\begin{gathered}
\text { Berechnet für } \\
\mathrm{C}_{9} \mathrm{H}_{8} \mathrm{~N}_{9} \mathrm{~S}
\end{gathered}
$$

$\mathbf{N}$
Gefunden 16,26 .

Das isomere $\mu$-Imido-n-Phenylthiazolin ist jedenfalls sehr schwer darzustellen, da die directe Phenylirung des Amidothiazols mittelst Jodbenzol nicht wohl möglich ist. Diese Base bot aber aber auch deshalb kein besonderes Interesse, weil sich deren Nitrosokörper jedenfalls gleich dem der entsprechenden methylirten Base normal verhalten, d. i. kein Condensationsproduct geliefert haben würde. 
Diphenylimidothiazolin,

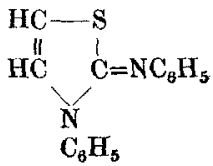

entsteht bei der Einwirkung von Dichloräther auf Diphenylthioharnstoff und wird aus dem sehr zerfliefslichen salzsauren Salz durch Kali abgeschieden. Es krystallisirt in kōrnigen Krystallen vom Schmelzpunkt $105^{0}$.

$0,1010 \mathrm{~g}$ Substanz lieferten 10,5 cbcm Stickgas bei $18^{\circ}$ und $720 \mathrm{~mm}$.

$$
\begin{array}{ccc} 
& \text { Berechnet für } & \text { Gefunden } \\
\mathrm{N} & \mathrm{C}_{15} \mathrm{H}_{12} \mathrm{~N}_{\mathbf{8}} \mathrm{K} & \\
11,11 & 11,36 .
\end{array}
$$

$\mu$-Phenyl-n-Nitrosoimidothiazolin<smiles></smiles>

fällt bei Zusatz einer concentrirten Natriumnitritlösung zu einer ebenfalls concentrirten, gut gekühlten Lösung des $\mu$-Phenylimidothiazolins in wenig Salzsäure sofort als nikrokrystallinisches Pulver aus. Abgeprefst und getrocknet bildet es gelbliche Krystalle vom Schmelzpunkt 58 ${ }^{\circ}$, besitzt ebenfalls einen charakteristischen würzigen Geruch, bleibt aber auch getrocknet nicht unverändert, sondern bräunt sich nach kurzer Zeit.

$0,0987 \mathrm{~g}$ Substanz lieferten $18,1 \mathrm{cbem}$ Stickgas bei $15^{0}$ und $722 \mathrm{~mm}$.

$$
\begin{gathered}
\text { Berechnet fïir } \\
\mathrm{C}_{9} \mathrm{H}_{7} \mathrm{~N}_{3} \mathrm{SO} \\
20,49
\end{gathered}
$$

Gefuuden

Wie schon erwähnt, weicht dieser phenylirte Nitrosokörper von dem analog constituirten Methylderivat erheblich ab. Wohl deshalb, weil die bei diesem letzteren eintretende Ringbildung unmöglich ist, verhält er sich weit mehr als echtes Nitrosamin. So wird er durch Alkalien nicht gelöst, durch Säuren aber ziemlich glatt in die Phenylbase zurückverwandelt. Vor allem wurde durch Salzsäure unter keinen Umständen ein schwer lösliches, salzsaures Salz gebildet, welches dem Chlor- 
hydrat des Thiazoltriazols entsprochen hätte. Beim Uebergiefsen des Nitrosokörpers mit 10 procentiger Salzsäure entweicht nur massenhaft salpetrige Säure und durch Kali wird die ursprüngliche Base vom Schmelzpunkt $124^{\circ}$ abgeschieden.

Auch auf das Diphenylimidothiazolin wirkt salpetrige Säure ein. Doch können hier nur Benzolwasserstoffe durch Nitrosyl substituirt werden, da ja das Dimethylimidothiazolin mit salpetriger Säure nicht reagirt. Es wurde deshalb von der Untersuchung dieser Nitrosokörper abgesehen.

\section{Zusammenfassung.}

1. Salpetrige Säure wirkt auf Amidothiazol ein unter Bildung eines zwischen Diazo- und Nitrosoverbindungen stehenden Körpers, den man als Diazothiazolhydrat oder Nitrosoimidothiazolin bezeichnen kann.

2. Bei der Einführung von Methylresten entstehen aus Amidothiazol zwei vom Imidothiazolin sich ableitende isomere Monomethylbasen und ein Dimethylderivat.

3. Durch salpetrige Säure entstelıen aus den isomeren Monomethylderivaten zwei isomere Nitrosamine; auf das Dimethylderivat wirlt salpetrige Säure nicht ein.

4. Das seitlich nitrosirte, im Kern methylirte Imidothiazolin ist ein echtes Nitrosamin, es geht durch schwache Reduction in das $n$-Methylthiazolinhydrazin über.

5. Das seitlich methylirte, im Kern nitrosirte Imidothiazolin weicht stark ab von den Nitrosaminen; durch Reduction entsteht kein Hydrazin. Salzsäure und Bromwasserstoffsäure bilden unter Wasseraustritt eine Anhydrobase, das Thiazoltriazol.

6. Von phenylirten Imidothiazolinen wurde das $\mu$-phenylirte und das diphenylirte Product dargestellt. Das Nitrosoderivat des ersteren liefert, abweichend von dem entsprechenden Körper der Methylreihe, keine Anhydrobase, sondern verhält sich wie ein echtes Nitrosamin.

(Geschlossen den 23. Juni 1891.) 